A Typology of Masspersonal Information Seeking Repertoires (MISR):

Global implications for political participation and subjective well-being

James H. Liu, Robert Jiqi Zhang, Roosevelt Vilar,

Massey University, New Zealand

Petar Milojev, Singidunum University, Serbia

Moh. Abdul Hakim, Universitas Sebelas Maret, Indonesia

Homero Gil de Zúñiga, University of Vienna \& Universidad Diego Portales, Chile

Sandy Schumann, University College London, UK

Dario Páez, University of the Basque Country, Spain \& Universidad Andrés Bello, Chile

May 17, 2020

RUNNING HEAD: Masspersonal Information Seeking Repertoires

Address Correspondence to:

Professor James Liu

School of Psychology

Massey University

Private Bag 102904

Auckland 0745, New Zealand

Email: J.h.liu@massey.ac.nz

This research was supported by Grant FA2386-15-1-0003 from the Asian Office of Aerospace Research and Development. 


\begin{abstract}
Masspersonal Information Seeking Repertoires are a person-centered method of gaining insight into the relationship between internet use, subjective well-being, and political participation. Through latent profile analysis, three person types were identified in two waves of stratified samples in 18 countries $(\mathrm{N}=8352)$. In accord with the "augmentation hypothesis", high levels of interpersonal contact and traditional mass media usage covaried with high internet use for the Highly Engaged type, that had highest political participation and life satisfaction, political knowledge, low depressive symptoms, but also high anxiety. The other two types fit the "displacement hypothesis", where internet-based media displaces traditional media and face-to-face communication. Compared to the Digitally Immersed, the Traditional repertoire was more knowledgeable and politically engaged, and had better wellbeing. Latent transition analysis showed these repertoires were stable over 6 months. Identifying different types of people with different information seeking styles clarifies mixed results on effects of online mass media use.
\end{abstract}

Keywords: internet usage; information seeking; media repertoires; masspersonal communication; displacement hypothesis; augmentation hypothesis; political participation; subjective well-being; social media; latent profile analysis 


\section{A Typology of Masspersonal Information Seeking Repertoires (MISR): \\ Global implications for political participation and subjective well-being}

The spread of online media — such as social media platforms, online news websites, and digital citizen-journalism — has changed informational landscapes around the world. People are increasingly immersed in information ecologies that afford them high choice in deciding how and whether to access information. Defined as "purposive seeking for information as a consequence of a need to satisfy some goal," (Wilson, 2000, p 49), human information seeking behavior is taking place in increasingly diverse ways. The purpose of this paper is to theorize human information seeking across a multiplicity of different communication channels, wherein each channel is contextualized within the individual as a whole, to better understand effects of mass media usage on the subjective well-being and political participation of different person types.

Given how rapidly information landscapes are changing, it is not surprising that empirical research on the impact of internet-based mass media on subjective well-being and political participation has produced mixed results. Classic early research by Kraut et al. (1998) introduced the "internet paradox", where increased internet usage led to less face-toface contact and increased loneliness a year later; but this failed to replicate in a follow-up (Kraut et al., 2002). Meta-analyses have been similarly inconsistent: Huang (2010) reported a small but significant negative correlation between internet use and psychological well-being in a meta-analysis of 40 studies, but failed to replicate this in a second meta-analysis (Huang, 2012). In the domain of political engagement, the results of Boulianne's $(2009,2015)$ metaanalyses were also complex: while there were far more positive effects than negative effects of social media use on political/civic participation, many of them became non-significant after imposing statistical controls 
The current study draws from several literatures in an attempt to build a framework to contextualize how a person's information seeking (and information acquisition) ${ }^{1}$ across a wide range of different sources impacts on their political participation and subjective wellbeing. The focus here is on the implications of information seeking patterns across multiple sources, rather than detailed investigation of the process of information seeking itself. We begin with Bolter and Grusin's (1999) assertion that "No medium today, and certainly no single media event, seems to do its cultural work in isolation from other media, any more than it works in isolation from other social and economic forces" (p. 15). We concur with Brikše (2003), who writes, "Analysis of an information environment is one way of recording and evaluating changes in societies, because various forms of communications do not replace each other or exclude former ones. Instead, they tend to co-exist and supplement one another," (p. 369). To examine such theoretical assertions empirically, we draw from the literature on mass media repertoires (Heeter, 1985), where internet-based media are not taken in isolation (e.g. as independent variables in a regression equation), but instead are analyzed holistically with other forms of media usage. Overall usage patterns are interpreted as mass media repertoires. Using factor analysis (Yuan, 2011), mixture modelling (Ksiazek, Malthouse, \& Webster, 2010), cluster analysis (Lee \& Yang, 2014), and factor analysis plus cluster analysis (Edgerly, Vraga, Bode, Thorson, \& Thorson, 2018), researchers have found that (1) usages of "old" and "new" media often cohere, so that a typical finding is "news avoiders", a category of people who do not engage much with news in any mass media, (2) in some studies there are "news omnivores", or people who engage with news across a range of mass media (Edgerly et al., 2018; Ksiazek et al., 2010; Yuan, 2011), and (3) in some studies,

\footnotetext{
${ }^{1}$ We do not go into such detail as to distinguish between purposive information seeking and incidental information acquisition as in Williamson's (1998) ecological model. Rather, we bundle these together within the concept of repertoires, using the term information "seeking" to emphasize the role of human agency.
} 
repertoires are differentiated between "old" versus "new" media (Edgerly et al., 2018; Lee \& Yang, 2014).

Conceptually, we seek to advance this literature by updating and enriching the measurement of media repertoires to include social information seeking. With the advent of interactive internet (Web 2.0) technologies, Reardon and Rogers' (1988) call to break down the "false dichotomy" between mass communications and interpersonal communications becomes even more relevant. Following this call, O’Sullivan and Carr (2018) advanced the concept of masspersonal communication as emerging from "observations of instances when (a) individuals use conventional mass communication channels for interpersonal communication, (b) individuals use conventional interpersonal communication channels for mass communication, and (c) individuals engage in mass communication and interpersonal communication simultaneously" (p. 1164). To elaborate on this concept, we include both mass media usages and interpersonal (i.e. social) information seeking in our measure, thereby re-conceptualizing repertoires as part and parcel of masspersonal communications, and the types of information seeking it provides in the digital age (see also Chadwick, Dennis, \& Smith, 2016, French \& Basarova, 2017; Holbert, Zeng \& Robinson, 2017).

A comparatively novel statistical method, Latent Profile Analysis (Hagenaars \& McCutcheon, 2002) is used to identify recurrent patterns of attributes of information seeking and group them into person-centered profiles (i.e. repertoires, or types). Using LPA signals a theoretical commitment to bottom-up hypothesis testing about what types of information seeking tendencies go together among what groups of people. This contrasts to a top-down approach, where researchers use some a priori conceptual scheme to decide what information seeking behaviors should be classified together (e.g. Bucy \& Groshek, 2018). LPA is similar to cluster analysis (used in previous publications in this area), but is able to provide fit statistics that allow more robust forms of hypothesis testing about the groups it identifies than 
is possible in cluster analysis. We go beyond the previous literature on repertoires by providing evidence of the longitudinal stability (and impact) of "masspersonal" information seeking repertoires.

As a person-based measure, LPA attempts to capture as much as it can about the entire person in its analysis. The quality of an LPA typology depends on the coherence of its input measures, and the stability and breadth of the typology that emerges. By combining mass media usages and social/interpersonal information seeking, we aim to produce a measure of information seeking for the digital age with breadth and stability over time. This is not a dynamic model of information seeking (e.g. Wilson, 1999). Nevertheless, this measure has potential to gain insight into the forms (types) a social and mass-media constructed version of the self might take, relevant to both communications theory (Bolter \& Grusin, 1999, chapters 15-17) and psychology (Bargh \& McKenna, 2004).

In summary, the current study furthers the task of integrating across communication channels and sources by focusing on the person rather than the message or medium. It aims to construct a typology where individuals' use of interpersonal/social communications to gain information is analyzed together with their usage of traditional and online mass media channels for news seeking. In this person-centered approach (Brandtzaeg, 2010; Johnson \& Kulpa, 2007), mass media usages and interpersonal communication patterns are treated as information seeking characteristics of different types of people.

\section{Plausible Patterns for a Typology of Masspersonal Information Seeking Repertoires}

Caplan (2003) suggests that some people may prefer computer-based communication to face to face communication because they think that it's easier, less risky, and is more exciting. Some of these people, who may be psychologically distressed to start with, or less socially skilled (Kraut et al., 2002), are thought to engage in compulsive internet-based 
behavior that reduces mental health. Following these theorists, Huang (2010) posited that the negative mental health outcomes result from the displacement of face-to-face relationships and social support with brittle or shallow internet-based interactions that fail to satisfy human needs, while taking up valuable time. He reported a small but significant negative correlation between internet use and psychological well-being in a first meta-analysis of 40 studies, but failed to replicate this finding in a second meta-analysis (Huang, 2012). A more recent metaanalysis of social networking and depression by Baker and Perez Algorta (2016) similarly reported a "complex relationship between online social networking and depression involving factors that may mediate or moderate this relationship" (p. 640).

However, Orben and Przbylski's (2019a) analyses of massive databases from the US and UK concluded that digital technology use was responsible for only $0.4 \%$ of the variation in adolescent well-being (about the same effect size for wearing glasses!). Their similarly massive longitudinal diary studies found "little substantive statistically significant and negative associations between digital screen engagement and well-being in adolescents" (p. 693, Orben \& Przbylski, 2019b). These researchers concluded that "social media effects are nuanced, small at best, reciprocal over time, gender specific, and contingent on analytic methods" (p. 10226, Orben, Dienlin, \& Przybylski, 2019). Underlining this point, a recent longitudinal study found no relationship between amount of time spent on social media and depression or anxiety for 500 adolescents over the course of 8 years (Coyne, Rogers, Zurcher, Stockdale, \& Booth, 2019).

But other important cross-sectional studies continue to report negative associations between internet-based mass media use and subjective well-being (e.g., Brailovskaia, Velten \& Margaf, 2019; Reinecke et al., 2017). Song et al.'s (2014) meta-analysis reported a positive association between Facebook use and loneliness; Marino, Gini, Vieno \& Spada's (2018) subsequent meta-analysis also showed a positive correlation ( $r=.34)$ between Facebook use 
and psychological distress. Meanwhile, Kelen, McRae and Grealish's (2019) systematic review found many studies reporting positive associations between social media use and mental health issues, like depressive symptoms and anxiety.

More consistent findings might be produced if researchers focused their attention on identifying a person type whose information seeking orients them towards using online media in a way that displaces traditional face-to-face relationships and mass media, rather than analyzing this variable in isolation. The person's information seeking repertoire would then function as the whole, within which the effects of a single variable are aggregated with correlated variables. The previous literature has shown that a single variable's effects are almost always moderated or mediated by other variables in a given context, thereby generating inconsistent findings across studies. In the LPA approach adopted here, we hold that one logical context within which to contextualize the functioning of any individual variable is the person, and that person's information seeking tendencies should be analyzed as a comprehensive whole.

Hypothesis 1 (Displacement). Latent Profile Analysis is able to identify two distinct repertoires of mass media usage and social information seeking: one where usages of internet-based mass media displace traditional mass media and face-to-face contact (Digitally Immersed Types), and another type that focuses more on traditional mass media and face-toface contact (Traditional Types).

Alternatively, online media might add to or hybridize with traditional media, where the people who like to read newspapers to access information also are more likely to seek internet news and not only talk to others face-to-face, but use instant messaging and social media to connect as well. This pattern is called an "omnivorous" news repertoire (Edgerly et al., 2018; Ksiazek et al., 2010; Yuan, 2011). It is also referred to as augmentation (e.g., Wellman, 2001; Valkenburg et al., 2006; Ellison, Steinfield, \& Lampe, 2007; Wellman, 
Haase, Witte, \& Hampton, 2001). In studies reporting augmentation, the more people engage in political activities offline, the more they also discuss political matters online, and vice versa. Thus, new media usage might not be as differentiated and "alternative" as some proponents of social media claim. An evolving hybrid culture based on a synergy of "old" and "new" mass media use might emerge (Chadwick, Dennis, \& Smith, 2016), consistent with theorizing about masspersonal communication (French \& Bazarova, 2017; O’Sullivan \& Carr, 2018).

Hypothesis 2 (Augmentation). Latent profile analysis is able to identify two distinct repertoires of mass media usage and social information seeking, an overall Highly Engaged Type versus a comprehensively low engagement type.

If both the augmentation and displacement hypotheses hold true, this would result in a classic $2 \times 2$ typology with four mass media usage/social information seeking repertoires, involving high versus low levels of traditional and online mass media use (and interpersonal communications). We test whether these usage patterns are consistent over a six month testretest period.

\section{The Relationship of Masspersonal Information Seeking Repertoires (MISR) with Political Participation and Subjective Well-being}

Exposure to news (compared to entertainment) through mass media typically increases political and civic engagement (Boulianne, 2009, 2015). This relationship can be magnified by the greater availability of viewing choices with digital mass media (Prior, 2005). With the advent of the internet, scholars have highlighted the symbiotic and often reinvigorating relationship for democracy between various forms of media use and exposure to news and public affairs. Gil de Zúñiga, Molyneux and Zheng (2014), for example, reported that social media news use had direct positive effects on offline political participation, and 
indirect effects on online political participation. Gil de Zúñiga and Valenzuela (2011) found that the relationships between online and offline network size and civic engagement were positive and similar in strength. When the internet is used for news and information seeking, it makes engagement easier, less costly, and potentially more diverse (Bennett \& Iyengar, 2012). This is consistent with the media repertoires literature, where people who are "news avoiders" participate less politically compared to other repertoire holders (Edgerly et al., 2018; Ksiazek et al., 2010; Lee \& Yang, 2014; Yuan, 2011).

Hypothesis 3 (High Information Seeking is associated with more political participation). Consistent with the logic of augmentation, people who have more face-to-face and online social contacts and frequently use both online and traditional mass media to get news, will report more political participation and have better political knowledge than people who are less engaged with information on- and off-line.

Finally, connecting critical domains of interest, there is evidence that active political participation can improve subjective well-being (Serrat, Villar, Giuliani, \& Zacarés, 2017).

Augmentation. Following the augmentation hypothesis (2), those highly engaged with others in daily life offline are also likely to engage in a healthy pattern of social engagements online (see Ellison, Steinfeld, \& Lampe, 2007; Wellman, 2001), sometimes combining them in novel ways (O'Sullivan \& Carr, 2018). On the other hand, people who are disengaged with others socially offline and carry a similar pattern online (i.e., low engagement or interacting with large numbers of strangers, see Bessiere, Kiesler, Kraut \& Boneva, 2008) are likely to be less well off in terms of subjective well-being (Caplan, 2003).

Hypothesis 4 (High Information Seeking is associated with better Subjective WellBeing): People who have a high level of social contact on- and offline and pay high levels of 
attention to mass media for news on- and off-line will report better life satisfaction and lower anxiety and depressive symptoms than people who are less engaged.

Displacement. Finally, we believe that a masspersonal repertoires approach to information seeking can clarify previously mixed results in this area, because people high in information seeking online but low in information seeking offline are likely to fall into a different person-type than people who are high in all forms of information seeking, as detailed below.

Displacement, conceptualized within a repertoires approach, does not work as an isolated behavior, but as information seeking tendencies of a person type. That is, some people may develop "a preference for online social interaction as an alternative to face to face communication because they perceive it to be less threatening and perceive themselves to be more efficacious when interacting with others online" (Caplan, 2003, p. 627); these preferences for online interaction as an alternative to face to face interaction can lead to problems that impact on subjective well-being, because they displace traditional, healthy interaction that leads to positive affect and/or social support (Huang, 2010). For the person type described in Hypothesis 4 previously, this should not be a problem because their use of online media augments (adds to) their tendencies to engage with information through other channels of communication as well.

Thus, our final and perhaps most interesting hypothesis relates to the patterns of subjective well-being for people who use internet-based media as an alternative to traditional forms of mass media. Here, the literature suggests that for some people, intensive use of the internet for communication can displace face-to-face interaction, increase social isolation, decrease social support and integration, and finally, provoke lower positive affect, less selfefficacy and self-esteem (Huang, 2010 but see Huang, 2012 for limitations). In contrast, 
people who use traditional media to engage in social interaction and news consumption will have better life satisfaction and lower symptoms of depression and anxiety than those who over-rely on online media, because their social interactions are less brittle, and more grounded (see Caplan, 2003; Kraut et al., 1998; Parks \& Floyd, 1996).

Hypothesis 5 (Traditional is better than Digitally Immersed for subjective well-being). Person types who use traditional mass media and have more social interactions offline will have better life satisfaction and lower anxiety and depressive symptoms than the person type who displaying a digitally immersed repertoire.

Edgerly et al.’s (2018) study on repertoires did not report consistent differences in political participation between Traditional types and the Digitally Immersed. But Traditionals were found to have more political knowledge in S. Korea (Lee \& Yang, 2014). Gil de Zúñiga, Weeks, \& Ardèvol-Abreu (2017) similarly found that people who rely on social media for news were less politically knowledgeable than those who purposively seek news in the USA. Given this lack of consensus, we do not form a hypothesis here.

\section{Masspersonal Information Seeking Repertoires (MISR) across Time and Countries}

Finally, we wanted to explore the stability of the Masspersonal Information Seeking Repertoires (MISR) over a 6-month test-retest period, and report prevalence across cultures. We believe that MISR are a relatively stable individual difference, as media usage preferences are associated with cohort effects (e.g., aging, Holt, Shehata, Strömbäck, \& Ljungberg, 2013) and some aspects of personality (e.g. openness, Weaver, 1991). We present the distribution of MISR across cultures, as well as reporting on country-level moderators. Previous studies have shown that access to and use of mass media are influenced by macrolevel factors such as information infrastructure and economic development (Chen \& Wellman, 2004). The availability and affordability of the internet in a society provides 
informational and networking opportunities which might determine the diversification of behavioral patterns for mass media users in that country (Brandtzæg, 2010; Wellman, 2012). Thus, the prevalence of a particular MISR, especially the more digitally immersed type, might be associated with the development of internet infrastructure in a country.

\section{Methods}

\section{Participants and Sampling Procedure}

The present study analyzed portions of data collected in a 2-wave international online survey. Wave 1 was in September 2015, and Wave 2 in March 2016; each wave involved 2 weeks of concurrent data collection. The total sample consisted of 8352 respondents who participated in both waves $\left(50.8 \%\right.$ women; $\left.M_{\text {age }}=46.33, S D=14.601\right)$ from 18 countries (Argentina, Brazil, Estonia, Germany, Indonesia, Italy, Japan, Korea, New Zealand, Philippines, Poland, Russia, Spain, Taiwan, Turkey, United Kingdom, Ukraine, United States). The survey was administrated by Nielsen, an international polling company that creates representative samples by using stratified quota sampling techniques (stratified by gender, age, and region of residence; see Gil de Zúñiga and Liu, 2017 for detailed information $^{2}$ ). Nielsen used email to contact prospective participants. Sample retention was assisted via three spaced email reminders to Wave 1 participants, together with variable incentives in different countries (administered by local panel providers partnering with Nielsen).

The measures used here are selected from a much larger overall study designed to examine mass media and social influences, trust, and their implications for social attitudes,

\footnotetext{
${ }^{2}$ China and Chile from Wave 1 were excluded, because China lacked democratic political participation measures, and Chile due to an administrative error that prevented connecting Wave 2 to Wave 1 participants.
} 
especially those towards the United States and its people. Many publications have resulted from this enterprise ${ }^{3}$.

\section{Measures}

Masspersonal Information Seeking Repertoires (MISR) were modelled using two components: 1) accessing news from various forms of mass media by asking "How often do you get news from..." the following mass media: $T V\left(\mathrm{M}_{\mathrm{w} 1}=5.56, \mathrm{SD}_{\mathrm{W} 1}=1.561 ; \mathrm{M}_{\mathrm{W} 2}=\right.$ $\left.5.61, \mathrm{SD}_{\mathrm{w} 2}=1.568\right)$, Printed Newspapers $\left(\mathrm{M}_{\mathrm{w} 1}=4.30, \mathrm{SD}_{\mathrm{w} 1}=1.871 ; \mathrm{M}_{\mathrm{w} 2}=4.16, \mathrm{SD}_{\mathrm{w} 2}=\right.$ 1.888), Radio $\left(\mathrm{M}_{\mathrm{w} 1}=4.15, \mathrm{SD}_{\mathrm{w} 1}=1.867 ; \mathrm{M}_{\mathrm{w} 2}=4.10, \mathrm{SD}_{\mathrm{w} 2}=1.864\right)$, Online News Sites $\left(\mathrm{M}_{\mathrm{w} 1}=5.07, \mathrm{SD}_{\mathrm{w} 1}=1.645 ; \mathrm{M}_{\mathrm{w} 2}=5.27, \mathrm{SD}_{\mathrm{w} 2}=1.579\right)$, Social Media $\left(\mathrm{M}_{\mathrm{w} 1}=4.05, \mathrm{SD}_{\mathrm{w} 1}=\right.$ 1.989; $\left.\mathrm{M}_{\mathrm{w} 2}=4.09, \mathrm{SD}_{\mathrm{w} 2}=1.980\right)$; and (2) interpersonal contact through various communication channels by asking "How often do you [meet face to face/talk over the phone/etc.] to stay in touch with their family and friends"; the following channels: Face to Face $\left(\mathrm{M}_{\mathrm{w} 1}=5.27, \mathrm{SD}_{\mathrm{w} 1}=1.333 ; \mathrm{M}_{\mathrm{w} 2}=5.23, \mathrm{SD}_{\mathrm{w} 2}=1.323\right)$, Phone $\left(\mathrm{M}_{\mathrm{w} 1}=5.12, \mathrm{SD}_{\mathrm{w} 1}=\right.$ $\left.1.402 ; \mathrm{M}_{\mathrm{w} 2}=5.07, \mathrm{SD}_{\mathrm{w} 2}=1.398\right)$, Email $\left(\mathrm{M}_{\mathrm{w} 1}=3.99, \mathrm{SD}_{\mathrm{w} 1}=1.726 ; \mathrm{M}_{\mathrm{w} 2}=3.95, \mathrm{SD}_{\mathrm{w} 2}=\right.$ 1.731), Video Chat $\left(\mathrm{M}_{\mathrm{w} 1}=3.02, \mathrm{SD}_{\mathrm{w} 1}=1.900 ; \mathrm{M}_{\mathrm{w} 2}=3.01, \mathrm{SD}_{\mathrm{w} 2}=1.872\right)$, Instant Messaging $\left(\mathrm{M}_{\mathrm{w} 1}=4.59, \mathrm{SD}_{\mathrm{w} 1}=1.869 ; \mathrm{M}_{\mathrm{w} 2}=4.56, \mathrm{SD}_{\mathrm{w} 2}=1.864\right)$. All were continuous variables assessed on a scale from 1 "never" to 7 "always". To minimize the potential influence of response tendency across cultures (Smith \& Fischer, 2008), each variable was group-centered by subtracting the national mean from raw scores.

In order to investigate the social and psychological associations of different MISR, a range of auxiliary variables were used. Basic demographics included self-reported measures of age (years), gender, and social status ("On a scale of 1 to 10 with 10 being people who are

\footnotetext{
${ }^{3}$ For a full publications listing, see https://www.dropbox.com/s/oko40j9uzzh1i8j/Digital\%20Influence\%20World\%20Project\%20Research\%20Out put.docx?dl=0
} 
the most well off in society, and 1 being the people who are the least well off, where would you describe your own position? 1 least well off, 10 most well off").

Three multi-choice items assessing political knowledge were included (i.e. who is the UN Secretary-General, what organizations monitor nuclear energy around the world, and understanding of the concept of global warming), and the total number of correct answers was used as a measure of participants' political knowledge.

Drawing on previous studies, political interest was assessed by two items: "How closely do you pay attention to information about what's going on in politics and public affairs?" and "How interested are you in information about what's going on in politics and public affairs?" on 7-point scales ranging from "not at all" to "very closely"/“a great deal" (Spearman-Brown coefficient $=.944$ in both Wave 1 and Wave 2). Political elaboration was measured by a 4-item scale (e.g., "I often find myself thinking about my conversations with other people about politics and public affairs after the discussion has ended"; Cronbach's $\alpha=$ .921 in Wave 1 and .930 in Wave 2). It was measured using 7-point Likert scales ranging from "disagree completely" to "agree completely". Finally, local/national voting was measured by "Usually, as far as you can recall, how often do you vote in local or statewide elections/ national or presidential elections?" on 7-point Likert scales ranging from "never" to "all the time".

A 5-item scale of Satisfaction with Life adapted from the Personal Well-being Index (PWI; Lau, Cummins, \& McPherson, 2005) was included (e.g., "All things considered, how satisfied are you these days with your life as a whole"; Cronbach's $\alpha=.865$ in Wave 1 and .869 in Wave 2). Anxiety was measured by an adapted 7-item GAD-7 scale (Spitzer, Kroenke, Williams, \& Löwe, 2006; e.g., "During the past two weeks, how often have you been bothered by feeling nervous, anxious, or on edge"; Cronbach's $\alpha=.938$ in Wave 1 and 
.944 in Wave 2). Depressive symptoms were measured by an adapted 2-item PHQ-2 scale (Kroenke, Spitzer, Williams, \& Löwe, 2009; e.g., "During the past two weeks, how often have you been bothered by having little interest or pleasure in doing things"; SpearmanBrown coefficient $=.861$ in Wave 1 and .871 in Wave 2). All these items were assessed on 7 point Likert scales ranging from "never" to "always".

Finally, two variables relevant to information overload (see Hargittai, Neuman, \& Curry, 2012; Ji, Ha, \& Sypher, 2014) were assessed: “About how many hours per day would you say that you are online?" (from 0-24hrs), and a self-reported measure of internet addition: "I am addicted to the internet" (7 point scale from completely disagree to completely agree). These were included to flesh out descriptions of the emergent repertoires.

\section{Statistical Analysis}

To identify distinct $M I S R$, we first conducted exploratory Latent Profile Analysis (LPA; Hagenaars \& McCutcheon, 2002) on the cross-sectional data within each wave separately. A set of models with different numbers of latent profiles (ranging from 2 to 5 profiles) were tested and compared to determine the optimal number of profiles. We used multiple criteria to decide the optimal model: the comparison of Information Criteria (AIC, aBIC), Entropy, and Lo-Mendell-Rubin testing procedure (LMR; Lo, Mendell, \& Rubin, 2001). Lower values of AIC and aBIC indicate better model fit, whereas higher values of Entropy (ranging from 0 to 1 ) indicate better model fit. The contribution and interpretability of identified repertoires was also considered in model selection.

The relationship between identified repertoires and auxiliary variables (i.e., demographics, well-being, and political participation) was assessed using a 3-step approach, following Asparouhov and Muthén's (2014) recommendation. Cohen's $\omega$ was calculated to 
indicate the strength of these associations, where $0.1,0.3$, and 0.5 correspond to small, medium, and large effect sizes, respectively (Cohen, 1988, p.224-227).

Then, Latent Transition Analysis (LTA; Collins \& Lanza, 2013) was conducted on the data from both waves simultaneously. LTA is a longitudinal extension of LPA, which assesses the stability or change of MISR by providing transition probabilities between profiles/repertoires across the two waves.

Finally, following Hanke et al. (2015), we used prevalence mapping to evaluate the distribution of MISR across countries based on the LTA model. All analyses were conducted in MPlus 7.31, using a MLR estimator (Maximum likelihood with robust standard errors).

\section{Results}

\section{Cross-sectional Latent Profile Analysis}

LPA was conducted on data from each wave separately. Fit indices are presented in Table 1. In Wave 1, increase in number of profiles led to decrease in AIC and aBIC. However, the LMR test suggested that loss of model fit from the 3-profile solution to 4profile solution was the least dramatic $(\mathrm{LMR}=1544.024, p=.001)$; and the 3-profile solution had the highest Entropy (0.767) among solutions. In Wave 2, the model fit results were similar to those in Wave 1: increase in number of profiles led to decrease in AIC and aBIC, but the LMR test suggested that the 4-profile solution did not significantly improve model fit compared to the 3-profile solution $(\mathrm{LMR}=1600.360, p=.110)$. The 3-profile solution also had good Entropy (0.761). Moreover, the 3-profile solution provided distinct and theoretically meaningful profiles in both waves, which was not characteristic of larger profile solutions ${ }^{4}$. Thus the 3 -profile solution was chosen as optimal

\footnotetext{
${ }^{4}$ A 4-profile solution that included a Low Engagement repertoire was found in Wave 1, but not in Wave 2.
} 
The three identified information seeking types are presented for Wave 1 in Figure 1. The identified solutions from each wave were almost identical similar in terms of structure and proportions, so the Wave 2 solution is not shown graphically. The first repertoire/profile (44.6\%, N=3724 in Wave 1, and 39.3\%, N=3272 in Wave 2) was characterized by relatively high scores on all indicator items, and is labelled "High Engagement". These people fit an augmentation hypothesis, where high internet-based media use co-varied with high traditional media usage.

The other two repertoires were both lower on almost all scores compared to the High Engagement Repertoire. The second repertoire was labelled as "Digitally Immersed" representing $13.5 \%(n=1129)$ in Wave 1 and $15.2 \%(n=1261)$ of the sample in Wave 2. The third repertoire was labelled "Traditional" - representing 41.9\% $(n=3498)$ in Wave 1 and 45.5\% ( $\mathrm{n}=3789)$ in Wave 2. As can be seen in Figure 1, the Traditional repertoire had higher frequencies of using traditional forms of news media (TV, newspapers, radio, but also online news) than the Digitally Immersed repertoire. On the other hand, the Digital repertoire had higher frequency of using news from social media. In terms of interpersonal contact, the Traditional repertoire had slightly higher frequencies of traditional means of contact (face to face, phone). The Digital repertoire had higher frequencies of use of digital news and contact (social media, email, video, IM). The identification of these latter two repertoires supports the displacement hypothesis, as the Digital and Traditional repertoires exhibited contrasting patterns, where the Digitals had comparatively low levels of face-to-face contact and traditional mass media usage compared to the Traditionals.

Thus, overall about $60 \%$ or so of participants fit with Hypothesis 1 (displacement $=$ Digital versus Traditional repertoires), and 40\% Hypothesis 2 (augmentation= High engagement repertoire). Results did not fit a neat $2 \times 2$ pattern. A low usage repertoire was 
missing, probably because of the longitudinal, online nature of the sample, and measurement indicators (including social/interpersonal information seeking, not just mass media).

\section{Relationships between MISR and other variables}

The 3-step approach yielded very similar cross-sectional results in both waves regarding the relationship between the identified MISR and auxiliary variables. To avoid repetition, results based on the cross-sectional Wave 1 data are reported in Table 2 . Wave 2 results can be found in supplementary materials (they were virtually the same). For demographics, small but significant differences in age were observed $\left(\chi^{2}=275.586, p<.001\right.$, $\omega=0.182)$, with the Traditional repertoire being oldest $(M=47.1)$, followed by the High Engagement repertoire (M=45.2). The Digitally Immersed repertoire was youngest $(M=39.4)$.

Significant but small differences according to gender were observed $\left(\chi^{2}=52.172, p<\right.$ $.001, \omega=.080$ ), with the Traditional repertoire having a higher proportion of men than the other two. Small but significant differences in self-reported social status were observed $\left(\chi^{2}=\right.$ 0.204, $p<.001, \omega=.204$ ), with the High repertoire having the highest self-reported social status, followed by Traditionals, and the Digital repertoire being lowest (see top of Table 2).

For political participation, significant but small differences were observed on political knowledge $\left(\chi^{2}=106.577, p<.001, \omega=.115\right)$, a medium-sized significant difference was found for political interest $\left(\chi^{2}=768.390, p<.001, \omega=.304\right)$, and small to medium differences for political elaboration $\left(\chi^{2}=547.704, p<.001, \omega=.256\right)$, local voting $\left(\chi^{2}=\right.$ 252.272, $p<.001, \omega=.175)$, and national voting $\left(\chi^{2}=214.726, p<.001, \omega=.161\right)$. On all these indicators, the High repertoire had the highest scores, followed by the Traditional repertoire. The Digital repertoire had the lowest scores. 
Overall, results fully supported Hypothesis 3: the High Engagement repertoire had more knowledge and was more politically engaged than the Digitally Immersed repertoire and the Traditional repertoire on every measure.

For subjective well-being, small to medium differences in Satisfaction with Life were observed $\left(\chi^{2}=540.556, p<.001, \omega=.255\right)$, with the High repertoire reporting the highest levels of life satisfaction, followed by the Traditional repertoire, then the Digital repertoire. Significant but very small differences in terms of self-reported anxiety were also observed $\left(\chi^{2}\right.$ $=69.329, p<.001, \omega=.091)$. Higher levels of anxiety were reported by the High repertoire and the Digital repertoire compared to the Traditional repertoire. Significant but very small differences in terms of self-reported depressive symptoms were also observed $\left(\chi^{2}=8.761, p\right.$ $<.001, \omega=.032$ ). The Digital repertoire reported higher levels of depressive symptoms compared to the High and Traditional repertoires.

Hypothesis 4 therefore received mixed support: the High repertoire had greater life satisfaction than the other repertoires, and had lower depressive symptoms than the Digital repertoire, but this group also had higher anxiety compared to the Traditional repertoire.

Hypothesis 5 was generally supported: the Traditional repertoire had lower anxiety, lower depressive symptoms, and was higher on satisfaction with life compared to the Digital repertoire. Confirming the displacement hypothesis, traditional forms of masspersonal information seeking were associated with better mental health and life satisfaction compared to being Digitally Immersed.

Cross-lagged associations using the 3-step approach, with MISR in Wave 1 as predictors, and auxiliary variables for Wave 2 as outcomes (see Table 3) yielded very similar results to those reported previously using cross-sectional analyses. The only exception was on Satisfaction with Life, where the Highly Engaged were more satisfied than other two 
repertoires, but there was no significant difference between the Traditionals and Digitals. Thus, the results reported were robust over a 6-month time lag.

One final, descriptive analysis on information overload showed that the High repertoire reported being online more hours per day than the Digital repertoire, who were higher than Traditionals. Both the High and Digital repertoires self-reported a higher level of internet addiction than Traditionals. There appear to be different ways of managing information overload: older Traditionals spend less time online, perhaps thereby experiencing less anxiety than the other information seeking types; but they also suffered a performance deficit on most other indicators compared to the Highly Engaged. The High Engagement repertoire was able to achieve high levels of performance across a range of indicators despite signs of information overload (see Hargittai et al., 2012 for subjective elements in information overload). Information overload hit the Digitally Immersed the hardest, because they allowed time on the internet to displace traditional ways of connecting with others.

\section{Latent Transition Analysis}

As the cross-sectional LPA yielded very similar 3-repertoire solutions across waves, an LTA model with the assumption of full longitudinal measurement invariance was specified. Specifically, the same latent profile indicators were constrained to be equal across the two waves in this model, and therefore are graphically identical to Figure 1 . The prevalence and transition probabilities are presented in the Table 4 . The top panel of the Table 4 shows the longitudinal prevalence of the three MISR was very similar to those identified in the cross-sectional LPAs. Over the course of 6 months, the proportion of the Digital repertoire increased from $14.8 \%$ in Wave 1 to $16.5 \%$ in Wave 2; the proportion of the traditional repertoire decreased from $44.0 \%$ in Wave 1 to $42.3 \%$ in Wave 2; the High repertoire comprised $41.2 \%$ in both Wave 1 and Wave 2 . The bottom panel shows the 
conditional probability of repertoires in Wave 2 given the repertoires in Wave 1 . The value on the diagonal indicates the proportion of individuals staying in the same repertoire. More than $85 \%$ of individuals stayed in the same repertoire over the course of 6 months. The most noticeable changes were $7.4 \%$ of individuals in the Digital repertoire in Wave 1 transitioned to Traditional in Wave 2; and 7.7\% of the Digital repertoire in Wave 1 transitioned to the High repertoire in Wave 2. This suggests that the MISR were relatively stable across 2 time points. Given that we have only two observation points, these data can say more about the stability of repertoires than likely reasons for transitions between them.

\section{Cross-cultural Analyses}

The results of prevalence mapping are presented visually for Wave 1 in Figure 2, and comprehensively for both Waves in Table 5. Less developed countries like the Philippines, Turkey, and Indonesia had a relatively low proportion of the Digital repertoire, while more developed countries like the USA and UK had high proportions of Digitals. Ukraine was the exception to the general rule that more Digitals were to be found in countries with a higher Human Development Index (HDI; UNDP, 2015). The fact that Ukraine was at war (with Russia) during the time of measurement could be responsible for it having such an unusually high proportion of the most vulnerable information seeking type in this study.

Interestingly, the High Engagement Repertoire was most common in developing countries Indonesia and Turkey, and lowest in Ukraine. It is difficult to ascertain much of a pattern on High Engagement from Figure 2; if anything, developing countries appeared to have a slightly higher proportion of the Highly Engaged compared to developed countries.

Overall, there appears a slight trend (with the glaring exception of Ukraine) for there to be more Digitals in higher HDI countries in each of the geographic zones. Similarly, there was a slight trend for the Traditional repertoire to be more common in more developed 
countries (but the Philippines violates this trend). An increase in the proportion of the Digital repertoire over time was observed across all countries except Estonia. This is likely an effect of the longitudinal (online) sampling frame.

Finally, multilevel analyses were conducted additionally to test potential crossnational variations on the relationship between MISR and auxiliary variables. Specifically, the two contrasting repertoire memberships were coded $(0=$ traditional and $1=$ digital $)$ as the predictor in multi-level analyses. Age, gender, and social status were included as control variables. Three country-level variables were included as country-level moderators in separate analyses of political participation and subjective well-being. They were HDI (UNDP, 2015), GDP per capita in 2015 (World Bank), and Global Connectivity Index (GCI; Huawei, 2015). Only one of the cross-level interactions was significant, and this was in accord with our prevalence mapping: Digitals reported lower political interest than Traditionals in countries with higher GDP per capita $(b=-.000, t=-2.038, p=.046)$. Thus, as a whole, the results we have reported were consistent across the 18 countries sampled.

\section{Discussion}

Results from a Latent Profile Analysis (LPA) of a two-wave online panel of 8352 individuals, representative of 18 countries in terms of age, gender, and region, provided insight into the relationships between media usage, interpersonal communications, political participation and subjective well-being. LPA was used to derive Masspersonal Information Seeking Repertoires (MISR), where patterns of media usage were combined with interpersonal communication tendencies to create a typology of information seeking repertoires. Model fit indices suggested that there are three ways of engaging with information, shared by people around the world with ready access to the internet: Highly Engaged, Traditional, and (least common) Digitally Immersed. 
MISRs contextualize the effects of individual mass media usage variables by locating them within a typology of people's overall patterns of information seeking. This is quite a different approach to information seeking than is current in information behavior studies (Savolainen, 2007; Wilson, 2000). Our current typology lacks input measures that focus attention on different stages and nuances in the dynamics of information processing (e.g. Williamson, 1998; Wilson, 1999). Its focus is limited to stable, self-reported patterns in using different communications channels for news and for keeping in touch. Although we chose to describe these as "information seeking repertoires", such emphasis on human agency (rooted in uses and gratifications theory, Rubin, 1993) might shortchange deeper psychological aspects of the types. There could be habit and/or compulsion involved, especially amongst the numerically small Digitally Immersed type, who might not be able to help themselves from displacing time better spent on face-to-face relationships, because of "fear of missing out" on information from the internet (Oberst et al., 2017).

Finding Digital and Traditional profiles supported Hypothesis 1, that internet-based information seeking can displace traditional ways of knowing. About $15 \%$ of the sample was classified as Digitally Immersed, whereas around 40\% were classified as Traditional. The Digitally Immersed were more likely to make use of instant messaging, social media and video to interact, compared to the Traditional type, who were more likely to get news from television, newspapers, radio, and to communicate face-to-face or by phone. Around 55-60\% of our global sample fit with the idea that the process of forming a new communications culture involves the "old" being displaced by the "new".

Associations of these repertoires with mental health confirmed Hypothesis 5. Traditional types reported less anxiety and lower depressive symptoms than the Digitally Immersed. Traditional types also reported higher satisfaction with life in the cross-sectional data, although the lagged association suggested this difference was not significant over time. 
In terms of political participation, results also suggested displacement: Traditionals reported more interest in politics, had more political elaboration, were more likely to vote, and had more factual knowledge about global political issues than the Digitally Immersed. However, the Traditional repertoire was older (by 7.5 years on average), had a higher proportion of men, and reported higher social status than the Digitally Immersed, so cohort effects (see Holt et al., 2013) are likely to have been embedded within these repertoires. Future research over a longer time period and with more observation points is required to unfold the deeper reasons behind the patterns observed.

In summary, if internet-based ways of engagement (e.g., instant messaging, video, and social media) displace traditional forms of engagement (e.g. face to face contact, and using traditional mass media to get news) across a wide range of information seeking indictors, then this is associated with worse mental health, and with having less political knowledge and participation. These results accord with the internet paradox first identified by Kraut et al. (1998) and meta-analyses by Huang (2010) and Song et al. (2014). The repertoires approach adopted here may function as a holistic alternative to the more analytical approach of regression analysis, where the impact of any one variable is likely to be moderated or mediated by other variables in some contexts, but not others. We found that across 18 cultures and over time, the Digitally Immersed person-type was associated with negative outcomes in terms of both subjective well-being and political participation compared to both Traditional and Highly Engaged person-types.

As a phenomenon, such displacement may occur at different rates around the world. The Digitally Immersed type is relatively frequent in countries like the USA, which is the most advanced state in terms of internet infrastructure according to the Global Connectivity Index (Huawei, 2015), and relatively low in developing countries like the Philippines, Turkey, and Indonesia. Given the limited country-level sample size $(N=18)$ in the present 
study, further studies are needed to explore how displacement is associated with macro-level factors.

What LPA adds is insight into the small effect sizes found in meta-analyses, and their inconsistency with other findings. In accord with Hypothesis 2, and the idea of a "news omnivore” (Edgerly et al., 2018; Ksiazek et al., 2010; Yuan, 2011), a Highly Engaged repertoire was identified, characterized by high values on every indicator, so that internetbased ways of knowing and interacting were combined with traditional forms of mass media consumption and social interaction. This person-type frequently engaged with others across all modes of communication, and scored positively on most associated variables. They thus showed augmentation (Hypothesis 4). LPA suggests that there is no one-to-one mapping between any one social media or internet usage variable and well-being, but rather, different strokes are configured to have different impact for different folks. Heavy internet usage might have positive benefits for some people.

This result shows that the proliferation of internet-based "new" media does not necessarily provoke a decrease in use of traditional media; for some people "old" and "new" communicative cultures are combined in a synergetic way (see Chadwick et al., 2016). These people could be more frequently engaged in masspersonal communication (O'Sullivan \& Carr, 2018). They might exert personal agency, both in how they choose to deal with mass media, and in how they engage with people. Future research could examine more precisely the content and purpose (see Lilleker \& Koc-Michalska, 2017) and detailed processes (see Williamson, 1998; Wilson, 2000) of information seeking by different person types, in different social environments.

The "Highly Engaged" pattern of high life satisfaction (and high self-reported social status) together with high anxiety and moderate depressive symptoms (mixed support for 
Hypothesis 4) could be considered as a characteristic adaptation for an information intensive age. Wealth of information can be good and bad: studies have shown that use of internet to follow rumors (Gosling \& Mason, 2015; Páez et al., 2019) is related to uncertainty and anxiety. Incivility is a regular feature of Web 2.0 information flows, which can provoke anger and hostility in return (Gervais, 2015). These instances of the internet-based amplification of negative information help explain the higher anxiety of the Highly Engaged, a repertoire also associated with more life satisfaction, political knowledge and participation (in accord with Hypothesis 3). Future research examining personality and value-based correlates over multiple observation points could ascertain what aspects of the self are enduringly connected to what information seeking tendencies (Bolter \& Grusin, 1999).

Conclusion. Three Masspersonal Information Seeking Repertoires (MISR) were found in large, representative, global samples. The Traditional type was oldest, had highest proportions of men, and was lowest on symptoms of depression and anxiety, with medium levels of life satisfaction, political participation and political knowledge. The Highly Engaged were highest on life satisfaction, self-reported social status, and anxiety, as well as political participation and knowledge. The Digitally Immersed were youngest and scored worse than the other two types on most indictors. It is the Digitally Immersed type that most clearly shows the dysfunctions of displacement, where internet-based activity is associated with less time spent on traditional communications and face-to-face relationships.

This pattern of results helps to explain why the relationship between internet usage and well-being and political participation is so variable and inconsistent across different studies: for some people (i.e., the Highly Engaged), internet-based media augment traditional media and thus internet usage has beneficial associations for political engagement and wellbeing. But for others (i.e. the Digitally Immersed), "new" media appears to displace "old" media and face-to-face communications, producing negative outcomes. To understand its 
consequences, internet use can be usefully contextualized as part of people's overall information seeking repertoires, and perhaps their deeper habits of society and mind. 


\section{References}

Aarts, S., Peek, S.T.M., \& Wouters, E.J.M. (2014). The relation between social network site usage and loneliness and mental health in community-dwelling older adults. International Journal of Geriatric Psychiatry, 30, 942-949.

Asparouhov, T., \& Muthén, B. (2014). Auxiliary variables in mixture modeling: Three-step approaches using M plus. Structural equation modeling: a multidisciplinary journal, 21(3), $329-341$.

Bagchi, K. K., Udo, G., Kirs, P. J., \& Choden, K. (2015). Internet use and human values: Analyses of developing and developed countries. Computers in Human Behavior, 50, 76-90.

Baker, D.A., Perez Algorta, G. (2016). The relationship between online social networking and depression: A systematic review of quantitative studies. Cyberpsychology, Behavior, and Social Networking, 19(11), 638-648. http://dx.doi.org/10.1089/cyber.2016.0206.

Bargh, J.A. \& McKenna, K.Y.A. (2004). The internet and social life. Annual Review of Psychology, 55, 573-590.

Bennett, W. L., \& Iyengar, S. (2008). A new era of minimal effects? The changing foundations of political communication. Journal of Communication, 58(4), 707-731.

Bessiere, K., Kiesler, S., Kraut, R., \& Boneva, B. S. (2008). Effects of Internet use and social resources on changes in depression. Information, Community \& Society, 11(1), 47-70.

Bolter, J.D., \& Grusin, R. (1999). Remediation: Understanding new media. Cambridge, MA: MIT Press.

Boulianne, S. (2009). Does internet use affect engagement? A meta-analysis of research. Political Communication, 26, 193-211. 
Brailovskaia, J., Velten, J., \& Margaf, J. (2019). Relationship between daily stress, depression symptoms, and facebook addiction disorder in Germany and in the United States. Cyberpsychology, Behavior, and Social Networking, 22 (9), 610-614. DOI: 10.1089/cyber.2019.0165

Brantzaeg, P.B. (2010). Towards a unified Media-User Typology (MUT): A meta-analysis and review of the research literature on media-user typologies. Computers in Human Behavior, 26, 940-956.

Brikše, I. (2003). The information environment: theoretical approaches and explanations. European Journal of Communication 19 (2): 368-415.

Bucy, E.G., \& Groshek, J. (2018). Empirical support for the media participation hypothesis: Trends across presidential elections, 1992-2012. New Media \& Society, 20(5), 1889-1909. Caplan, S.E. (2003). Preference for online social interaction: A theory of problematic internet use and psychosocial well-being. Communication Research, 30(6), 625-648.

Chadwick, A., Dennis, J., \& Smith, A.P. (2016). Politics in the age of hybrid media: power, systems, and media logics. In Bruns, A. et al. (eds.) The Routledge Companion to Social Media and Politics. New York: Routledge, pp. 7 - 22

Chen, W. \& Wellman, B. (2004). The global digital divide - within and between countries. IT \& Society, 1(7), 18-25.

Cohen, J. (1988). Statistical Power Analysis for the Behavioral Sciences. Hillsdale, NJ: L. Erlbaum Associates.

Collins, L. M., \& Lanza, S. T. (2013). Latent class and latent transition analysis: With applications in the social, behavioral, and health sciences (Vol. 718): John Wiley \& Sons. 
Coyne, S.M., Rogers, A.A, Zurcher, J.D., Stockdale, L., \& Booth, M. (2019). Does time spent using social media impact mental health?: An eight year longitudinal study. Computers in Human Behavior, 104, https://doi.org/10.1016/j.chb.2019.106160.

Edgerly, S., Vraga, E. K., Bode, L., Thorson, K., \& Thorson, E. (2018). New media, new relationship to participation? A closer look at youth news repertoires and political participation. Journalism \& Mass Communication Quarterly, 95, 192-212.

Ellison, N.B., Steinfield, C., \& Lampe, C. (2007). The benefits of Facebook “'friends:”' Social capital and college students' use of online social network sites. Journal of ComputerMediated Communication, 12, 1143-1168.

French, M., \& Bazarova, N.N. (2017). Is anybody out there? Understanding masspersonal communication through expectations for response across social media platforms. Journal of Computer-Mediated Communication, 22, 303-309.

Bryan T. Gervais (2015) Incivility online: Affective and behavioral reactions to uncivil political posts in a web-based experiment, Journal of Information Technology \& Politics, 12(2), 167-185, DOI: 10.1080/19331681.2014.997416

Gil de Zúñiga, H., \& Liu, J.H. (2017). Second screening politics in the social media sphere: Advancing research on dual screen use in political communication with evidence from 20 countries. Journal of Broadcasting \& Electronic Media. 61(2) 193-219.

Gil de Zúñiga, H., Molyneux, L., \& Zheng, P. (2012). Social media, political expression, and political participation: Panel analysis of lagged and concurrent relationships. Journal of Communication, 64, 612-634. 
Gil de Zúñiga, H., \& Valenzuela, S. (2011). The mediating path to stronger citizenship: Online and offline networks, weak ties, and civic engagement. Communication Research, 38, $397-421$.

Gil de Zúñiga, H., Weeks, B., Ardèvol-Abreu, A. (2017). Effects of the News-Finds-Me perception in communication: Social media use implications for news seeking and learning about politics, Journal of Computer-Mediated Communication, 22(3), 105123, https://doi.org/10.1111/jcc4.12185

Gosling, S.D., \& Mason, W. (2015). Internet research in psychology. Annual Review of Psychology, 66, 877-902. DOI: 10.1146/annurev-psych-010814-015321

Hagenaars, J.A., \& McCutcheon, A.L. (2002). Applied latent class analysis. Cambridge: Cambridge University Press.

Hanke, K., Liu, J,H., Sibley, C., Paez, D., Gaines, S.P., Jr, Moloney, G., et al., (2015). "Heroes" and "Villains" of world history across cultures. PLOS-ONE, 10(2): e0115641. doi:10.1371/journal.pone.0115641

Hargittai, E., Neuman, W., \& Curry, O. (2012). Taming the information tide: Perceptions of information overload in the American home. The Information Society, 28(3), 161-173.

Heeter, C. (1985). Program selection with abundance of choice: A process model. Human Communication Research, 12, 126-152. doi:10.1111/j.1468-2958.1985.tb00070.x

Holbert, R. L., Zeng, C., \& Robinson, N. W. (2017). Adopting an integrated behavioral model approach to the study of news media exposure: A focus on experiential and instrumental attitudes toward politics. Mass Communication and Society, 20(4), 573-593. 
Holt, K., Shehata, A., Strömbäck, J., \& Ljungberg, E. (2013). Age and the effects of news media attention and social media use on political interest and participation: Do social media function as leveller?. European Journal of Communication, 28(1), 19-34.

Huang, C. (2010). Internet use and psychological well-being: a meta-analysis.

Cyberpsychology Behavior, and Social Networking, 13(3), 241-249.

Huang, C. (2012). Internet use and psychological well-being. In Z. Yan (Ed.), Encyclopedia of Research on Cyber Behavior (pp. 302-314). Hershey, PA, USA: Information Science Reference, IGI Global.

Huawei. (2015). Global Connectivity Index. Retrieved from https://www.huawei.com/minisite/gci/en/country-rankings.html Ji, Q., Ha, L., \& Sypher, U. (2014). The role of news media use and demographic characteristics in the prediction of information overload. International Journal of Communication, 8, 699-714.

Johnson, G. M., \& Kulpa, A. (2007). Dimensions of online behavior: Toward a user typology. CyberPsychology \& Behavior, 10(6), 773-780.

Keles, B., McCrae, N., \& Grealish, A. (2019). A systematic review: the influence of social media on depression, anxiety and psychological distress in adolescents, International Journal of Adolescence and Youth, DOI: 10.1080/02673843.2019.1590851

Kraut, R., Kiesler, S., Boneva, B., Cummings, J., Helgeson, V., \& Crawford, A. (2002). Internet Paradox Revisited. Journal of Social Issues, 58(1), 49-74. https://doi.org/10.1111/1540-4560.00248 
Kraut, R., Patterson, M., Lundmark, V., Kiesler, S., Mukopadhyay, T., \& Scherlis, W. (1998). Internet paradox: A social technology that reduces social involvement. American Psychologist, 53(9), 1017-1031.

Kroenke, K., Spitzer, R. L., Williams, J. B., \& Löwe, B. (2009). An ultra-brief screening scale for anxiety and depression: the PHQ-4. Psychosomatics, 50(6), 613-621.

Ksiazek, T. B., Malthouse, E. C., \& Webster, J. G. (2010). News seekers and avoiders: Exploring patterns of total news consumption across media and the relationship to civic participation. Journal of Broadcasting \& Electronic Media, 54(4), 551-568.

Lau, A. L., Cummins, R. A., \& McPherson, W. (2005). An investigation into the crosscultural equivalence of the Personal Well-being Index. Social Indicators Research, 72, 403430.

Lee, H., \& Yang, J. (2014). Political knowledge gaps among news consumers with different news media repertoires across multiple platforms. International Journal of Communication, $8,597-617$.

Lilleker, D. G., \& Koc-Michalska, K. (2017). What drives political participation? Motivations and mobilization in a digital age. Political Communication, 34(1), 21-43.

Lo, Y., Mendell, N.R., \& Rubin, D.B. (2001). Testing the number of components in a normal mixture. Biometrika, 88(3), 767-778.

Marino, C., Gini, G., Vieno, A. \& Spada, M.A. (2018). The associations between problematic Facebook use, psychological distress and well-being among adolescents and young adults: A systematic review and meta-analysis, Journal of Affective Disorders, 226, 274-281. https://doi.org/10.1016/j.jad.2017.10.007 
Oberst, U., Wegmann, E., Stodt, B., Brand, M., Chamarro, A. (2017). Negative consequences from heavy social networking in adolescents: the mediating role of fear of missing out. Journal of Adolescence, 55, 51-60.

Orben, A., Dienlin, T., \& Przybylski, A.K. (2019). Social media’s enduring effect on adolescent life satisfaction. PNAS, 116, 10226-10228.

Orben, A., \& Przybylski, A. K. (2019a). The association between adolescent well-being and digital technology use. Nature Human Behaviour, 3(2), 173.

Orben, A., \& Przybylski, A. K. (2019b). Screens, teens, and psychological well-being: Evidence from three time-use-diary studies. Psychological science, 30(5), 682-696.

O’Sullivan, P.B., \& Carr, C.T. (2018). Masspersonal communication: A model bridging the mass-interpersonal divide. New Media \& Society, 20(3), 1161-1180.

Páez, D., Delfino, G., Vargas-Salfate, S., Liu, J.H., Gil de Zuñiga, H., Khan, S., \& Garaigordobil, M. (2019). A longitudinal study of the effects of internet use on subjective well-being: Effects of direct and online social contact among representative samples in 19 nations. Media Psychology. DOI: 10.1080/15213269.2019.1624177

Parks, M. R., \& Floyd, K. (1996). Making friends in cyberspace. Journal of ComputerMediated Communication, 1, 80-97.

Prior, M. (2003). Any good news in soft news? The impact of soft news preference on political knowledge. Political Communication, 20(2), 149-171.

Prior, M. (2005). News vs. entertainment: How increasing media choice widens gaps in political knowledge and turnout. American Journal of Political Science, 49(3), 577-592. 
Reardon, K. K. \& Rogers, E. M. (1988). Interpersonal versus mass media communication: A false dichotomy. Human Communication Research, 15, 284-303.

Reinecke, L., Aufenanger, S., Beutel, M. E., Dreier, M., Quiring, O., Stark, B., ... Müller, K. W. (2017). Digital stress over the life span: The effects of communication load and internet multitasking on perceived stress and psychological health impairments in a German probability sample. Media Psychology, 20(1), 90-115. doi:10.1080/15213269.2015.1121832 Rubin, A.M. (1993) Audience activity and media use, Communications Monographs, 60(1), 98-105, DOI: 10.1080/03637759309376300

Savolainen, R. (2007). Information behavior and information practice: Reviewing the “Umbrella Concepts” of information-seeking studies. The Library Quarterly: Information, Community, Policy, 77(2), 109-132.

Serrat, R., Villar, F., Giuliani, M. F., \& Zacarés, J. J. (2017). Older people’s participation in political organizations: The role of generativity and its impact on well-being. Educational Gerontology, 43(3), 128-138.

Song, H., Zmyslinski-Seelig, A., Kim, J., Drent, A., Victor, A., Omori, K., \& Allen, M. (2014). Does Facebook make you lonely? A meta analysis. Computers in Human Behavior, $36,446-452$.

Spitzer, R. L., Kroenke, K., Williams, J. B., \& Löwe, B. (2006). A brief measure for assessing generalized anxiety disorder: the GAD-7. Archives of Internal Medicine, 166(10), 1092-1097.

Smith, P. B., \& Fischer, R. (2008). Acquiescence, extreme response bias and culture: A multilevel analysis. In F. J. R. van de Vijver, D. A. van Hermert, \& Y. H. Poortinga (Eds.), Multilevel analysis of individuals and cultures (pp. 285-314). New York: Lawrence Erlbaum. 
United Nations Development Programme. (2016). Human Development Report 2016.

Retrieved from

https://sustainabledevelopment.un.org/content/documents/22212015_human_development_re port.pdf

Valkenburg, P.M., \& Peter, J. (2009). Social consequences of the internet for adolescents: A decade of research. Current Directions in Psychological Science, 18(1), 1-5.

Weaver, J. B., III (1991). Exploring the links between personality and media preferences. Personality and Individual Differences, 12, 1293-1299.

Wellman, B. (2001). Computer networks as social networks. Science, 293(5537), 2031-2034.

Wellman, B., Haase, A.Q., Witte, J., \& Hampton, K. (2001). Does the internet increase, decrease, or supplement social capital? American Behavioral Scientist, 45(3), 436-455.

Williamson, K. (1998). Discovered by chance: The role of incidental information acquisition in an ecological model of information use. Library \& Information Science Research, 20, 2340.

Wilson, T. D.(1999). Models in information behaviour research. Journal of Documentation, 55(3), 249-270.

Wilson, T.D. (2000). Human information behavior. Informing Science, 3(2), 49-55.

World Bank. GDP per capita. Retrieved from https://data.worldbank.org/indicator/NY.GDP.PCAP.CD?end=2016\&most_recent_year_desc $=$ true $\&$ start $=2016$

Yuan, E. (2011). News consumption across multiple media platforms: A repertoire approach. Information, Communication \& Society, 14(7), 998-1016. 
Table 1. Information Criteria, the LMR statistics, and Entropy for cross-sectional LPAs.

\begin{tabular}{lllll}
\hline & AIC & aBIC & LMR $(\mathrm{p})$ & Entropy \\
\hline Wave 1 & & & & \\
2 repertoires & 305173.075 & 305292.497 & $8702.227(<.001)$ & 0.703 \\
3 repertoires & 302302.581 & 302464.379 & $2863.664(<.001)$ & 0.767 \\
4 repertoires & 300765.014 & 300969.187 & $1544.024(.001)$ & 0.751 \\
5 repertoires & 299583.202 & 299829.750 & $1191.814(<.001)$ & 0.756 \\
Wave 2 & & & & \\
2 repertoires & 303378.705 & 303498.019 & $9205.968(<.001)$ & 0.708 \\
3 repertoires & 300589.391 & 300751.042 & $2783.283(<.001)$ & 0.761 \\
4 repertoires & 298994.914 & 299198.902 & $1600.360(.110)$ & 0.774 \\
5 repertoires & 297676.265 & 297922.591 & $1327.281(.025)$ & 0.750 \\
\hline
\end{tabular}

Note: AIC - Akaike Information Criterion; aBIC - adjusted Bayesian Information Criterion; LMR - Lo-Mendell-Rubin statistic (p value). 
Table 2. Cross-sectional associations between Masspersonal Information Seeking Repertoires (MISR) and dependent variables based on the data in Wave 1.

\begin{tabular}{|c|c|c|c|c|c|}
\hline & Digital Repertoire (A) & Traditional Repertoire (B) & High Repertoire (C) & Approximate $\chi^{2}$ & $\omega$ \\
\hline \multicolumn{6}{|l|}{ Demographics } \\
\hline Age & 39.427 в,С & $47.109 \mathrm{~A}, \mathrm{C}$ & $45.190 \mathrm{~A}, \mathrm{~B}$ & $275.586^{* * *}$ & 0.182 \\
\hline Gender ( $\%$ men $)$ & 0.449 в & $0.557_{\mathrm{A}, \mathrm{C}}$ & $0.457_{\mathrm{B}}$ & $52.172^{* * *}$ & 0.080 \\
\hline Social Status & 5.014 B,C & $5.151_{\mathrm{A}, \mathrm{C}}$ & $5.838 \mathrm{~A}, \mathrm{~B}$ & $346.600^{* * *}$ & 0.204 \\
\hline \multicolumn{6}{|l|}{ Political Participation } \\
\hline Political Knowledge & $1.639 \mathrm{~B}, \mathrm{C}$ & $1.909 \mathrm{~A}, \mathrm{C}$ & $1.969 \mathrm{~A}, \mathrm{~B}$ & $106.577^{* * *}$ & 0.115 \\
\hline Political Interest & 3.758 B,C & $4.499 \mathrm{~A}, \mathrm{C}$ & $5.045 \mathrm{~A}, \mathrm{~B}$ & $768.390^{* * *}$ & 0.304 \\
\hline Political Elaboration & $3.491 \mathrm{~B}, \mathrm{C}$ & $3.617 \mathrm{~A}, \mathrm{C}$ & $4.314_{\mathrm{A}, \mathrm{B}}$ & $547.704^{* * *}$ & 0.256 \\
\hline Local Voting & 4.533 B,C & $5.458 \mathrm{~A}, \mathrm{C}$ & $5.715_{\mathrm{A}, \mathrm{B}}$ & $252.272^{* * *}$ & 0.175 \\
\hline Nation Voting & $4.792 \mathrm{~B}, \mathrm{C}$ & $5.625 \mathrm{~A}, \mathrm{C}$ & $5.872_{\mathrm{A}, \mathrm{B}}$ & $214.726^{* * *}$ & 0.161 \\
\hline \multicolumn{6}{|l|}{ Well-being } \\
\hline Life Satisfaction & $4.477_{\mathrm{B}, \mathrm{C}}$ & $4.609 \mathrm{~A}, \mathrm{C}$ & $5.133 \mathrm{~A}, \mathrm{~B}$ & $540.556^{* * *}$ & 0.255 \\
\hline Anxiety & $3.230 \mathrm{~B}$ & $3.023 \mathrm{~A}, \mathrm{C}$ & 3.289 в & $69.329^{* * * *}$ & 0.091 \\
\hline Depression & $3.018 \mathrm{~B}, \mathrm{C}$ & $2.861 \mathrm{~A}$ & $2.886 \mathrm{~A}$ & $8.761^{*}$ & 0.032 \\
\hline \multicolumn{6}{|l|}{ Internet Usage } \\
\hline Hours online & $6.137 \mathrm{C}$ & $5.977 \mathrm{C}$ & $6.576 \mathrm{~A}, \mathrm{~B}$ & $32.225^{* * *}$ & 0.063 \\
\hline Internet addiction & $3.551_{\mathrm{B}}$ & $3.431_{\mathrm{A}, \mathrm{C}}$ & $3.641_{\mathrm{B}}$ & $25.966^{* * *}$ & 0.056 \\
\hline
\end{tabular}

Note. The mean values for each repertoire are presented. Analyses of continuous variables were run using the DCON procedure, while analysis on categorical variables were run using DCAT procedure in Mplus.

Subscripts indicate repertoires that are significantly different at $\mathrm{p}<.05$. Effect size was calculated with the formula $\omega=(\chi 2 / \mathrm{N})^{1 / 2}$.

$* p<.05, * * p<.01, * * * p<.001$ 
Table 3. Lagged associations between Masspersonal Information Seeking Repertoires (MISR) in Wave 1 and dependent variables in Wave 2.

\begin{tabular}{|c|c|c|c|c|c|}
\hline & Digital Repertoire (A) & Traditional Repertoire (B) & High Repertoire (C) & Approximate $\chi^{2}$ & $\omega$ \\
\hline \multicolumn{6}{|l|}{ Political Participation } \\
\hline Political Knowledge & $1.774 \mathrm{~B}, \mathrm{C}$ & $2.021 \mathrm{~A}, \mathrm{C}$ & $2.066 \mathrm{~A}, \mathrm{~B}$ & $77.678^{* * *}$ & 0.101 \\
\hline Political Interest & $3.838 \mathrm{~B}, \mathrm{C}$ & $4.560_{\mathrm{A}, \mathrm{C}}$ & $5.077_{\mathrm{A}, \mathrm{B}}$ & $667.625^{* * *}$ & 0.291 \\
\hline Political Elaboration & $3.670_{\mathrm{B}, \mathrm{C}}$ & $3.792 \mathrm{~A}, \mathrm{C}$ & $4.115 \mathrm{~A}, \mathrm{~B}$ & $182.154^{* * *}$ & 0.152 \\
\hline Local Voting & $4.668_{\mathrm{B}, \mathrm{C}}$ & $5.556 \mathrm{~A}, \mathrm{C}$ & $5.766 \mathrm{~A}, \mathrm{~B}$ & $208.976^{* * *}$ & 0.163 \\
\hline Nation Voting & $4.867 \mathrm{~B}, \mathrm{C}$ & $5.723 \mathrm{~A}, \mathrm{C}$ & $5.882 \mathrm{~A}, \mathrm{~B}$ & $179.474^{* * *}$ & 0.151 \\
\hline \multicolumn{6}{|l|}{ Well-being } \\
\hline SWL & $4.520_{\mathrm{C}}$ & $4.598_{\mathrm{C}}$ & $5.055 \mathrm{~A}, \mathrm{~B}$ & $377.136^{* * *}$ & 0.213 \\
\hline Anxiety & 3.249 в & $3.000 \mathrm{~A}, \mathrm{C}$ & 3.296 в & $86.264^{* * *}$ & 0.102 \\
\hline Depression & $3.042_{\mathrm{B}, \mathrm{C}}$ & $2.870 \mathrm{~A}$ & $2.900 \mathrm{~A}$ & $10.373^{* *}$ & 0.035 \\
\hline \multicolumn{6}{|l|}{ Internet Usage } \\
\hline Internet addiction & $3.477_{\mathrm{B}}$ & $3.018_{\mathrm{A}, \mathrm{C}}$ & $3.474_{\mathrm{B}}$ & $128.508^{* * *}$ & 0.126 \\
\hline
\end{tabular}


Table 4. LTA results of 3-repertoire solution for MISR

\begin{tabular}{lccr}
\hline & Digital & Traditional & High \\
\hline Prevalence of latent profiles & & & \\
Wave 1 & $14.8 \%$ & $44.0 \%$ & $41.2 \%$ \\
Wave 2 & $16.5 \%$ & $42.3 \%$ & $41.2 \%$ \\
\multicolumn{4}{c}{} \\
Transition probabilities (Rows for Wave 1 and Columns for Wave 2) \\
Digital & $85.0 \%$ & $7.4 \%$ & \\
Traditional & $5.8 \%$ & $88.4 \%$ & $5.7 \%$ \\
High & $3.8 \%$ & $4.9 \%$ & $91.3 \%$ \\
\hline
\end{tabular}

Note: transition probabilities were based on the estimated model. 
Table 5. Proportions of Masspersonal Information Seeking Profiles (MISR) across countries/societies (sorted based on the ascending proportion of the Digitally Immersed profile in Wave 1)

\begin{tabular}{|c|c|c|c|c|c|c|}
\hline Country & $\begin{array}{l}\text { Digital } \\
\text { W1 }\end{array}$ & $\begin{array}{l}\text { Digital } \\
\text { W2 }\end{array}$ & $\begin{array}{l}\text { Traditional } \\
\mathrm{W} 1\end{array}$ & $\begin{array}{l}\text { Traditional } \\
\text { W2 }\end{array}$ & $\begin{array}{l}\text { High } \\
\text { W1 }\end{array}$ & $\begin{array}{l}\text { High } \\
\text { W2 }\end{array}$ \\
\hline Philippines & 0.07 & 0.11 & 0.50 & 0.46 & 0.43 & 0.43 \\
\hline Turkey & 0.09 & 0.11 & 0.44 & 0.44 & 0.47 & 0.45 \\
\hline Indonesia & 0.10 & 0.13 & 0.41 & 0.41 & 0.49 & 0.47 \\
\hline Italy & 0.12 & 0.15 & 0.42 & 0.40 & 0.46 & 0.46 \\
\hline Taiwan & 0.12 & 0.14 & 0.49 & 0.46 & 0.39 & 0.40 \\
\hline Brazil & 0.13 & 0.15 & 0.42 & 0.40 & 0.45 & 0.46 \\
\hline Poland & 0.13 & 0.16 & 0.43 & 0.40 & 0.44 & 0.44 \\
\hline Argentina & 0.14 & 0.18 & 0.41 & 0.40 & 0.45 & 0.43 \\
\hline Spain & 0.14 & 0.17 & 0.48 & 0.42 & 0.38 & 0.42 \\
\hline Germany & 0.15 & 0.16 & 0.46 & 0.45 & 0.39 & 0.39 \\
\hline Estonia & 0.16 & 0.16 & 0.43 & 0.42 & 0.41 & 0.42 \\
\hline S.Korea & 0.16 & 0.18 & 0.43 & 0.41 & 0.42 & 0.41 \\
\hline Japan & 0.16 & 0.18 & 0.44 & 0.43 & 0.40 & 0.39 \\
\hline UK & 0.16 & 0.17 & 0.46 & 0.44 & 0.38 & 0.39 \\
\hline Russia & 0.16 & 0.17 & 0.42 & 0.42 & 0.42 & 0.42 \\
\hline $\mathrm{NZ}$ & 0.17 & 0.19 & 0.46 & 0.44 & 0.37 & 0.37 \\
\hline USA & 0.20 & 0.21 & 0.44 & 0.43 & 0.36 & 0.36 \\
\hline Ukraine & 0.31 & 0.33 & 0.40 & 0.37 & 0.30 & 0.31 \\
\hline
\end{tabular}




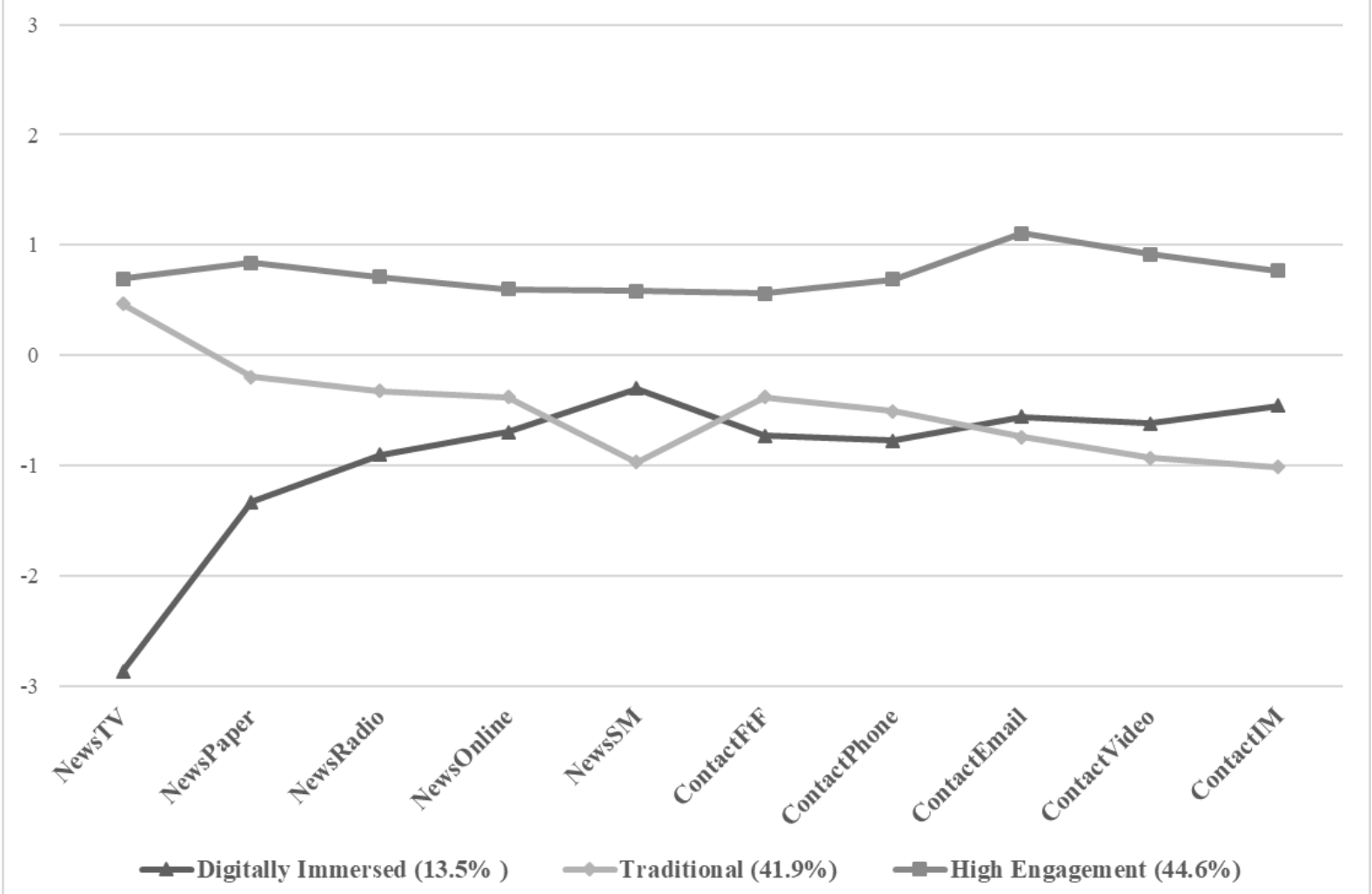

Figure 1. The identified Masspersonal Information Seeking Repertoires (MISR) based on Latent Profile Analysis on the cross-sectional data from Wave 1 


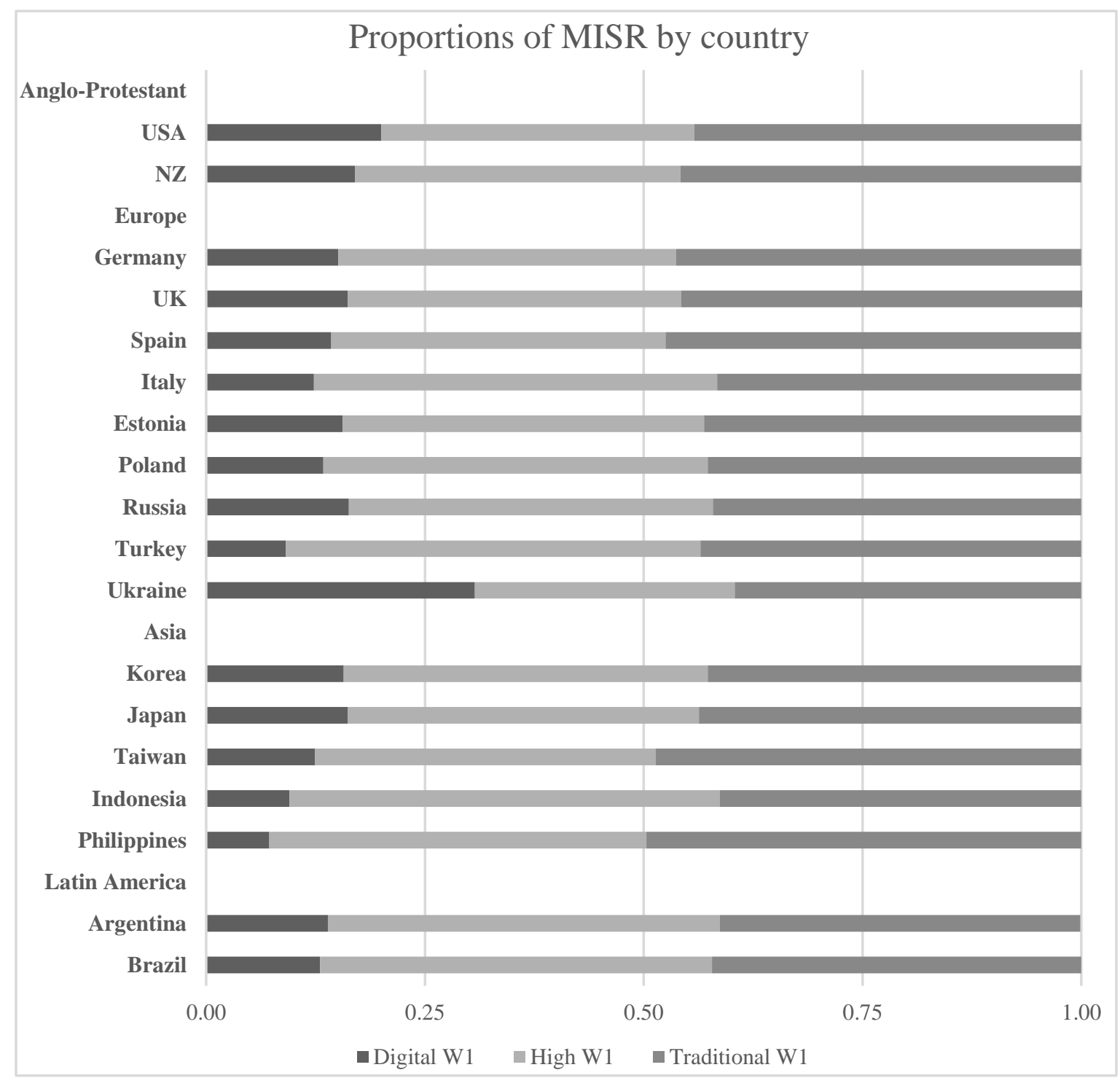

Figure 2. Proportions of Masspersonal Information Seeking Repertoires (MISR) by country in Wave 1 (sorted in descending order by Human Development Index in 2015 within region) 